\title{
Methylation of the RIN3 Promoter is Associated with Transient Ischemic Stroke/Mild Ischemic Stroke with Early Cognitive Impairment
}

\author{
Meng Miao (1) \\ Fang Yuan ${ }^{2}$ \\ Xiaotian $\mathrm{Ma}^{3}$ \\ Haiming Yang ${ }^{\prime}$ \\ Xiang Gao' \\ Zhengyu Zhu (iD ${ }^{4}$ \\ Jianzhong $\mathrm{Bi}^{4}$ \\ 'Department of Neurology, Qilu Hospital \\ (Qingdao), Cheeloo College of Medicine, \\ Shandong University, Qingdao, Shandong, \\ 266035, People's Republic of China: \\ ${ }^{2}$ Department of Health Care, Qilu \\ Hospital (Qingdao), Cheeloo College of \\ Medicine, Shandong University, Qingdao, \\ Shandong, 266035, People's Republic of \\ China; ${ }^{3}$ Department of Medicine \\ Experimental Center, Qilu Hospital \\ (Qingdao), Cheeloo College of Medicine, \\ Shandong University, Qingdao, Shandong, \\ 266035, People's Republic of China; \\ ${ }^{4}$ Department of Neurology, The Second \\ Hospital, Cheeloo College of Medicine, \\ Shandong University, Jinan, Shandong, \\ 250033, People's Republic of China
}

Correspondence: Zhengyu Zhu; Jianzhong $\mathrm{Bi}$

Department of Neurology, The Second Hospital, Cheeloo College of Medicine, Shandong University, Jinan, Shandong, 250033, People's Republic of China $\mathrm{Tel}+86$ I7660083488; +86I5I53I78III Email zhuzhengyu_I@hotmail.com; bjz@sdu.edu.cn
Background: Early cognitive impairment after transient ischemic stroke (TIA)/mild ischemic stroke (MIS) is common but easily overlooked. It has been demonstrated that DNA methylation plays a significant role in cognitive impairment and ischemic stroke. Furthermore, it has been reported that the RIN3 gene influences transportation of the amyloid $\beta$-protein. However, to our knowledge, there has been no research related to correlations between RIN3 methylation and early-onset cognitive impairment after TIA/ MIS. Therefore, this study aimed to investigate this relationship in TIA/MIS patients.

Methods: This study include 28 control subjects and 84 patients with TIA/MIS who were evaluated within 7 days of TIA/MIS onset using four single-domain cognitive scales. In addition, DNA methylation of whole blood was tested. RIN3 methylation was compared between TIA/MIS and control groups and between TIA/MIS patients with early cognitive impairment and those without early cognitive impairment. Clinical variables and RIN3 methylation sites with statistical differences were then used to construct a predictive model. Results: Hypomethylation of the RIN3 gene was observed in the whole blood of TIA/MIS patients relative to healthy controls. Furthermore, patients with early cognitive impairment after TIA/MIS had hypomethylation of RIN3 relative to those without early cognitive impairment.

Conclusion: RIN3 methylation is strongly associated with TIA/MIS and TIA/MIS with early cognitive impairment. It is possible to influence the disease process by methylation via appropriate lifestyle and clinical interventions, and methylation of RIN3 gene sites may predict the occurrence of TIA/MIS with early cognitive impairment.

Keywords: amyloid B-protein, cognitive impairment, hypomethylation, ischemic stroke, transportation

\section{Introduction}

Ischemic stroke, one of the most common cerebral vascular diseases, can lead to neurological and cognitive impairment and can also accelerate cognitive disorders. Cognitive impairment can also occur in the early phase after a transient ischemic stroke (TIA) or a mild ischemic stroke (MIS). Since the dyskinetic symptoms of TIA/MIS are mild and short-lived, cognitive impairment due to TIA/MIS is easily overlooked, and the cognitive level of patients after onset of TIA/MIS is not routinely assessed in clinical practice. Currently, the specific pathogenesis of early cognitive impairment after TIA/MIS is unclear and requires further research. 
It has been demonstrated that the incidence of early cognitive impairment after TIA/MIS can be as high as $32 \%-70 \% .^{1-4}$ Cognitive impairment is common in the acute phase of TIA/MIS and is more common within 7 days after TIA/MIS. Of note, the recovery of cognition in these patients does not parallel the resolution of somatic motor symptoms. Also, the acute phase of cognitive impairment is highly correlated with persistent future cognitive decline. ${ }^{2}$

DNA methylation, an important form of epigenetics, plays a significant role in several neurodegenerative pathologies, including Alzheimer's disease (AD) and parkinsonism. Abnormal methylation of several genes has also been observed in ischemic cerebrovascular disease. The RIN3 gene, located on chromosome 14 , acts as a stimulating factor to stabilise the transportation of GTP-RAB5 to endosomes at the plasma membrane. This process is associated with cellular endocytosis and has a negative effect on endocytosis of the amyloid $\beta$-protein $(\mathrm{A} \beta),{ }^{5,6}$ which plays a role in the onset of both $\mathrm{AD}$ and vascular cognitive impairment (VCI). Studies have shown that the RIN3 gene is highly expressed in $\mathrm{AD}^{7}$ and that this gene is hypomethylated in the whole blood of patients with early-onset $\mathrm{AD}{ }^{8}$

Based on these previous studies, we hypothesised that abnormal expression of the RIN3 gene would impact the occurrence of cognitive impairment. Furthermore, we hypothesized that methylation, the most important manifestation of epigenetics, would affect expression of the RIN3 gene. To our knowledge, there is no existing research on the correlation between RIN3 gene methylation and early-onset cognitive impairment after TIA/MIS. Therefore, this study aimed to determine if hypomethylation of the RIN3 gene was associated with TIA/MIS with early cognitive impairment.

In this study, we examined whole-blood methylation levels in three groups, including TIA/MIS patients with early cognitive impairment, TIA/MIS patients without cognitive impairment, and non-infarcted control subjects, in order to investigate the correlation between wholeblood RIN3 methylation levels and TIA/MIS with early cognitive impairment. In addition, we aimed to identify significant predictive factors for construction of predictive models and for development of interventions.

\section{Materials and Methods}

\section{Participants}

A total of 84 patients (age $<75$ years) with TIA/MIS were prospectively recruited from the Department of Neurology at the Qilu Hospital of Shandong University in Qingdao, China between June 2019 and July 2020. Mild stroke was defined as an acute cerebral infarction with a National Institutes of Health Stroke Scale (NIHSS) score of less than 5, and TIA was defined as a sudden focal neurological deficit in the brain, spinal cord, or retina lasting less than $24 \mathrm{~h}$, which results from atherosclerotic vascular causes and is not associated with an acute cerebral infarction. All TIA/MISs were recorded using the Trial of Org 10172 in acute stroke treatment (TOAST) classification system and confirmed using cranial computed tomography (CT) and/or magnetic resonance imaging (MRI). Patients were evaluated within 7 days of TIA/MIS onset using four single-domain cognitive scales: ${ }^{9-16}$ the Boston Naming Test (BNT) for language (abnormality: $\leq 21.5$ points; adjusted value: $\leq 19.5$ points if the education period was $\leq 9$ years and $\leq 21.5$ points if the education period was $>9$ years), the Auditory Verbal Learning Test (AVLT) for memory (abnormality: $<5$ points after $5 \mathrm{~min}$ ), and the Trail Making Test (TMT)-A for visuospatial function (abnormality: $\geq 98.5 \mathrm{~s}$; adjusted value: $\geq 80.5$ s for patients aged $\leq 64$ years, $\geq 90.5 \mathrm{~s}$ for those aged $64-74$ years, and $\geq 90.5 \mathrm{~s}$ for those aged $\geq 75$ years, with a maximum time of $150 \mathrm{~s}$ ), and TMT-B for executive function (abnormality: $\geq 188.5 \mathrm{~s}$; adjusted value: $\geq 150.5 \mathrm{~s}$ for patients aged $\leq 64$ years, $\geq 165.5 \mathrm{~s}$ for those aged $64-74$ years, and $\geq 199.5 \mathrm{~s}$ for those aged $\geq 75$ years, with a maximum time of $300 \mathrm{~s}$ ). Cognitive impairment was determined by more than one $(\geq 1)$ scale that has abnormal results. All cognitive screening were performed by two neurologists with at least 5 years of experience. Exclusion criteria included (1) stroke mimics; (2) age $>75$ years; (3) cerebral haemorrhage, degenerative disease, tumour, or severe hepatic or renal insufficiency; and (4) inability to complete cognitive scales cooperatively because of dysphasia or hearing or visual impairment. The control group (28 patients) included healthy volunteers or patients without TIA/cerebral infarction suffering from dizziness or headaches recruited during the same period; all accepted cranial $\mathrm{MRI} / \mathrm{CT}$ images and cerebrovascular screenings were used to exclude TIA/MIS. This study was approved by the Ethics Committee of the Qilu Hospital of Shandong University in Qingdao, China. All patients provided written informed consent for participation in this study.

\section{DNA Extraction and Quality Control}

Fasting blood samples were drawn using EDTA tubes, and whole-blood DNA was extracted using kits (Tiangen Biotech, Beijing, China). The quality of the DNA was detected by a NanoDrop 2000 (NanoDrop technologies, 
Wilmington, DE, USA), which required a concentration of $\geq 20 \mathrm{ng} / \mu \mathrm{L}$ and a total sample purity of $\geq 400 \mathrm{ng}$.

\section{CpG Island Selection}

$\mathrm{CpG}$ islands located in the proximal promoter of the RIN3 gene were selected for measurement according to the following criteria: (1) 200-bp minimum length; (2) $50 \%$ or higher GC content; and (3) 0.60 or higher ratio of observed/expected dinucleotide $\mathrm{CpG}$. Two regions from $\mathrm{CpG}$ islands of the RIN3 gene were selected and sequenced.

\section{Bisulfite Conversion and Multiplex Amplification}

DNA methylation levels were analysed using MethylTarget $^{\circledR}$ (Genesky Biotechnologies Inc., Shanghai, China), an NGS-based multiple targeted $\mathrm{CpG}$ methylation analysis method. Specifically, the genomic regions of interest were analysed and transformed into bisulfiteconverted sequences using GeneCpG software. Polymerase chain reaction (PCR) primer sets were designed using Methylation Primer software from bisulfate-converted DNA. The PCR primers for RIN3_26 were as follows: forward, 5'-GTATATTTGTTAGGA ATGTGGAGGAG-3'; reverse, 5'-AAAAAAAAATC TTCCACTTAACTTAAAACC- $3^{\prime}$. The PCR primers for RIN3_27 were as follows: forward, 5'TTAGTGTTTGGGTAGGGTTTAGG-3'; reverse, 5'AAACCCAACCCCRAACAA-3'. Genomic DNA (400 ng) was subjected to sodium bisulfite treatment using the EZ DNA Methylation ${ }^{\mathrm{TM}}$-GOLD Kit (ZYMO, CA, USA), according to the manufacturer's protocols. Multiplex PCR was performed using optimised primer set combinations. A $20-\mu \mathrm{L}$ PCR reaction mixture was prepared for each reaction, which included 10x reaction buffer (TaKaRa, Dalian, China), $25 \mathrm{mM}$ of $\mathrm{Mg}^{2+}, 2.5 \mathrm{mM}$ of dNTP, 1 $\mu \mathrm{M}$ of each primer, $5 \mathrm{U}$ of HotStarTaq polymerase (TaKaRa, Dalian, China), and $1 \mu \mathrm{L}$ of template DNA. The cycling program included $95^{\circ} \mathrm{C}$ for $2 \mathrm{~min} ; 11$ cycles of $95^{\circ} \mathrm{C}$ for $20 \mathrm{~s}, 62^{\circ} \mathrm{C}$ for $40 \mathrm{~s}$ with a decreasing temperature step of $0.5^{\circ} \mathrm{C}$ per cycle, and $72^{\circ} \mathrm{C}$ for $1 \mathrm{~min}$; and 24 cycles of $95^{\circ} \mathrm{C}$ for $20 \mathrm{~s}, 62^{\circ} \mathrm{C}$ for $30 \mathrm{~s}, 72^{\circ} \mathrm{C}$ for $1 \mathrm{~min}$, and $72^{\circ} \mathrm{C}$ for $1 \mathrm{~min}$.

\section{Index PCR}

PCR amplicons were diluted and amplified using indexed primers. Specifically, a $20-\mu \mathrm{L}$ mixture was prepared for each reaction comprised of $5 \mathrm{x}$ reaction buffer (TaKaRa,
Dalian, China), $2.5 \mathrm{mM}$ of dNTP, $10 \mu \mathrm{M}$ of F primer, 4 $\mu \mathrm{M}$ of index primer, $0.2 \mu \mathrm{L}$ of Herculase ${ }^{\circledR}$ II Fusion DNA polymerase (Agilent Technologies, CA, USA), $2 \mu \mathrm{L}$ of diluted template, and $\mathrm{ddH} 2 \mathrm{O}$. The cycling program included $95^{\circ} \mathrm{C}$ for $2 \mathrm{~min}$ followed by 11 cycles of $95^{\circ} \mathrm{C}$ for $20 \mathrm{~s}, 65^{\circ} \mathrm{C}$ for $30 \mathrm{~s}, 72^{\circ} \mathrm{C}$ for $30 \mathrm{~s}$, and $72^{\circ} \mathrm{C}$ for $3 \mathrm{~min}$. PCR amplicons (170 bp-270 bp) were separated by agarose electrophoresis and purified using the TIANGEN Gel Extraction kit (TIANGEN, Beijing, China).

\section{Sequencing}

Libraries from different samples were quantified and pooled together followed by sequencing on the Illumina HiSeq platform according to the manufacturer's protocols. Sequencing was performed using a $2 \times 150$-bp paired-end mode.

\section{Data Analysis}

Fast Length Adjustment of SHort reads (FLASH) is an accurate and fast tool used to merge paired-end reads. ${ }^{17}$ FASTQ to FASTA format was then processed using the Fastx toolkit (http://hannonlab.cshl.edu/fastx toolkit/ index.html). Reads in FASTA format were mapped to the targeted bisulfite genome (hg19) by Blast. ${ }^{18}$ Unmapped reads were filtered, and mapped reads with a coverage greater than $90 \%$ and an identity greater than $90 \%$ were considered effective reads and used for the following statistics. The sequencing depth for each amplicon per sample was calculated by blasting the effective reads against the targeted genomic region. Reads less than 10fold were removed, and the overall sequencing depth for each sample was evaluated. Methylation and haplotypes were analysed using Perl script.

\section{Statistical Methods}

$\mathrm{R}$ software was used for statistical analysis. Continuous data are presented as mean \pm standard deviation $(\bar{x} \pm s)$, and categorical data are expressed as numbers of cases and percentages ( $\mathrm{n}[\%])$. Student's $t$-test was used for continuous variables with normal distribution and homogeneous variance to compare observations between both study groups, and the Wilcoxon test was used otherwise. The test of proportions, including the K-square test and CochranArmitage trend test, was applied to determine if there was a statistically significant difference in attribute percentages between data sets. The K-square test was used for nominal categorical variables. The Cochran-Armitage trend test was used for ordinal categorical variables. Single-factor logistic 

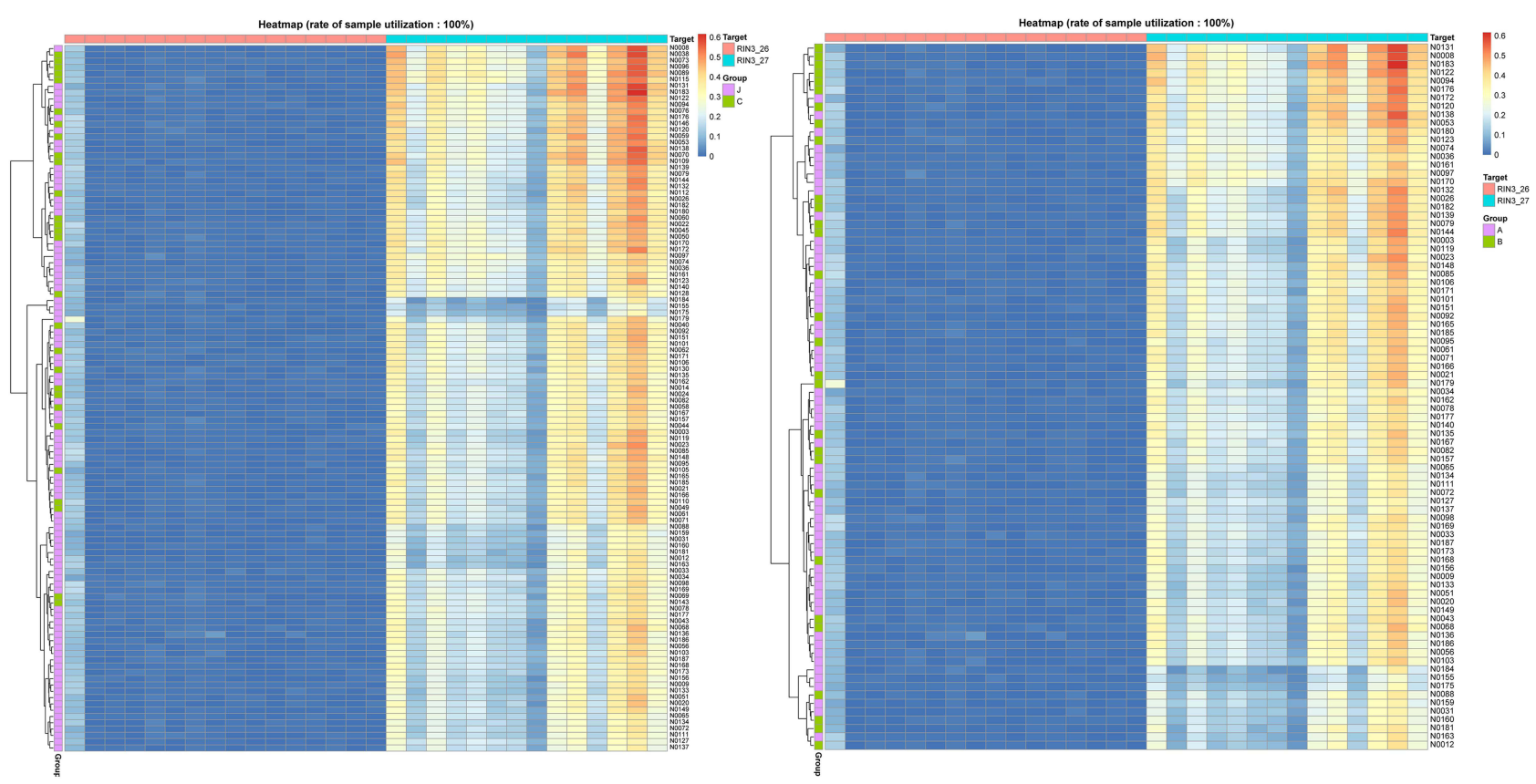

Figure I Shows a heatmap based on the methylation levels of $C_{p}$ G sites in all samples in groups J, C, A, and B. Each row is a sample, and each column is a CpG site. Each cell represents the relative methylation level at $C_{p} G$ sites in the corresponding row of samples. The colour gradient reflects the change in methylation level, with a tendency toward blue indicating a lower methylation level and a tendency toward red indicating a higher methylation level. The similarities between samples' methylation levels are indicated by the order of the rows, with adjacent rows indicating more similar overall methylation levels in the represented samples.

regression and multi-factor logistic regression analyses were performed to identify independent variables for TIS/MIS with early cognitive impairment. We screened variables using a stepwise regression analysis to construct predictive models and plot ROC curves. A p-value $<0.05$ was considered statistically significant.

\section{Results}

In this study, we tested whole-blood RIN3 methylation in 112 subjects (Figure 1), including 84 patients with TIA/ MIS in the case group (group J); 55 patients had early cognitive impairment (group A), whereas 29 participants did not (group B). Moreover, there were 28 participants in the control group (group $\mathrm{C}$ ). Basic patient clinical information are shown in Tables 1 and 2.

\section{Differences Between Patients with TIA/ MIS and Healthy Controls in Terms of Total RIN3 Methylation Level}

By sequencing methylation in the target regions (Table 3) in the 84 TIA/MIS and 28 controls, we found that the overall methylation level of the RIN3 gene was significantly lower in the TIA/MIS group than in the control group (Groupdiff $=-0.02, P<0.001)$ (Figure 2), even after adjusting for age and gender (Adj. $P<0.001$ ) (Table 4$)$.

\section{Total RIN3 Methylation Levels in Patients with TIA/MIS with and without Cognitive Impairment}

The overall methylation level of the RIN3 gene was significantly lower in the cognitive impairment group $(\mathrm{n}=55)$ than in the non-cognitive impairment group $(n=29)$ (Groupdiff $=-0.013, P=0.01$ ) (Figure 3), even after adjusting for age, gender, education, CHD, and DWM (Adj. $P=0.044$ ) (Table 5).

\section{Differences in Differentially Methylated Sites Between Patients with Transient Ischemic Stroke/Mild Ischemic Stroke and Healthy Controls}

Whole blood RIN3 gene methylation levels were significantly different between patients with TIA/MIS and controls. We evaluated differences in methylation levels at different sites between both groups and found statistically significant differences at several sites (S26-146, 
Table I Clinical Information on the Transient Ischemic Stroke/ Mild Ischemic Stroke and Control Groups

\begin{tabular}{|c|c|c|c|}
\hline & $\begin{array}{l}\text { TIA/MIS Group } \\
(n=84)\end{array}$ & $\begin{array}{l}\text { Control Group } \\
(n=28)\end{array}$ & $P$ value \\
\hline Age (years) $(\bar{x} \pm s)$ & $61.94 \pm 8.03$ & $57.64 \pm 9.00$ & 0.016 \\
\hline Females (n [\%]) & $26(31.0)$ & $15(53.6)$ & 0.054 \\
\hline Education (years) & & & 0.004 \\
\hline$\leq 6$ & 22 & 1 & \\
\hline$\leq 12$ & 51 & 19 & \\
\hline$>12$ & 11 & 8 & \\
\hline Diabetes mellitus (n [\%]) & $34(40.5)$ & $8(28.6)$ & 0.575 \\
\hline Hypertension (n [\%]) & $62(73.8)$ & $18(64.3)$ & 0.622 \\
\hline Hyperlipemia (n [\%]) & $22(26.2)$ & $12(42.9)$ & 0.035 \\
\hline $\begin{array}{l}\text { Coronary heart disease } \\
\text { (n [\%]) }\end{array}$ & $16(19.0)$ & $6(21.4)$ & 0.425 \\
\hline Alcohol (n [\%]) & $33(39.3)$ & $6(21.4)$ & 0.137 \\
\hline Smoking (n [\%]) & $35(41.7)$ & $9(32.1)$ & 0.503 \\
\hline \multicolumn{4}{|l|}{ Abnormal scale } \\
\hline BNT (n [\%]) & $16(19.0)$ & & \\
\hline $\operatorname{AVLT}(\mathrm{n}[\%])$ & $43(51.2)$ & & \\
\hline TMT-A (n [\%]) & $39(46.4)$ & & \\
\hline TMT-B (n [\%]) & $38(45.2)$ & & \\
\hline Fazekas PV & & & $<0.001$ \\
\hline 0 (n [\%]) & $\mathrm{I}(\mathrm{I} .2)$ & $10(35.7)$ & \\
\hline I (n [\%]) & $43(51.2)$ & $17(60.7)$ & \\
\hline 2 (n [\%]) & $27(32.1)$ & I (3.6) & \\
\hline 3 (n [\%]) & $13(15.5)$ & $0(0)$ & \\
\hline Fazekas DWM & & & $<0.001$ \\
\hline 0 (n [\%]) & $7(8.3)$ & $12(42.9)$ & \\
\hline I (n [\%]) & $44(52.4)$ & $14(50.00)$ & \\
\hline 2 (n [\%]) & $26(31.00)$ & $2(7.1)$ & \\
\hline 3 (n [\%]) & $7(8.3)$ & $0(0)$ & \\
\hline LDL (mmol/L) & $2.81 \pm 1.00$ & $3.12 \pm 1.02$ & 0.088 \\
\hline $\mathrm{HDL}(\mathrm{mmol} / \mathrm{L})$ & $1.10 \pm 0.24$ & $1.20 \pm 0.31$ & 0.074 \\
\hline $\mathrm{HCY}(\mu \mathrm{mol} / \mathrm{L})$ & $14.15 \pm 7.19$ & $12.45 \pm 6.01$ & 0.247 \\
\hline $\mathrm{UA}(\mu \mathrm{mol} / \mathrm{L})$ & $310.93 \pm 84.17$ & $339.37 \pm 97.59$ & 0.457 \\
\hline Vitamin $B / 2(p g / m L)$ & $411.61 \pm 251.58$ & $476.30 \pm 191.32$ & 0.057 \\
\hline
\end{tabular}

Notes: In the TIA/MIS group, 81 patients underwent low-density lipoprotein (LDL) testing, 80 underwent high-density lipoprotein (HDL) testing, 75 underwent homocysteine (HCY) testing, 72 underwent uric acid (UA) testing, and 63 underwent vitamin BI2 testing. In the control group, 27 participants underwent LDL and HDL testing, 25 underwent $\mathrm{HCY}$ testing, 27 underwent $\mathrm{UA}$ testing, and 20 underwent vitamin $\mathrm{B} / 2$ testing. Abbreviations: PV, periventricular; DWM, deep white matter.

$\mathrm{S} 27-25,29,36,38,40,58,61,96,111,116,127,144$, 146 , and 152, $P<0.05$ ), even after adjustment for age and gender. All of these sites showed hypomethylation
Table 2 Clinical Information on the Cognitive-Impairment and Con-Cognitive-Impairment Groups

\begin{tabular}{|c|c|c|c|}
\hline & $\begin{array}{l}\text { Cognitive } \\
\text { Impairment } \\
\text { Group }(n=55)\end{array}$ & $\begin{array}{l}\text { Non-Cognitive } \\
\text { Impairment } \\
\text { Group n=29) }\end{array}$ & $P$ value \\
\hline Age (years) $(\bar{x} \pm s)$ & $63.47 \pm 6.89$ & $59.03 \pm 9.16$ & 0.029 \\
\hline Female (n [\%]) & $20(36.4)$ & $6(20.7)$ & 0.219 \\
\hline $\begin{array}{l}\text { Education (years) } \\
\quad \leq 6 \\
\leq 12 \\
>12\end{array}$ & $\begin{array}{l}20 \\
31 \\
4\end{array}$ & $\begin{array}{l}2 \\
20 \\
7\end{array}$ & 0.001 \\
\hline Diabetes mellitus ( $[\%]$ ) & $21(38.2)$ & $13(44.8)$ & 0.722 \\
\hline Hypertension (n [\%]) & $38(69.1)$ & $24(82.8)$ & 0.145 \\
\hline Hyperlipemia (n [\%]) & $14(25.5)$ & $8(27.6)$ & 1 \\
\hline $\begin{array}{l}\text { Coronary heart disease } \\
\text { (n [\%]) }\end{array}$ & $14(25.5)$ & $2(6.9)$ & 0.077 \\
\hline TOAST & & & 0.077 \\
\hline $\begin{array}{l}\text { Large aryery } \\
\text { atheroscierosis (n [\%]) }\end{array}$ & $26(47.3)$ & $7(24.1)$ & \\
\hline $\begin{array}{l}\text { Small artery } \\
\text { occulusion (n [\%]) }\end{array}$ & $29(52.7)$ & $22(75.9)$ & \\
\hline Alcohol (n [\%]) & $22(40.0)$ & $11(37.9)$ & I \\
\hline Smoking (n [\%]) & $24(43.6)$ & II (37.9) & 0.786 \\
\hline $\begin{array}{l}\text { Abnormal scale } \\
\text { BNT (n [\%]) } \\
\text { AVLT (n [\%]) } \\
\text { TMT-A (n [\%]) } \\
\text { TMT-B (n [\%]) }\end{array}$ & $\begin{array}{l}16(29.1) \\
43(78.2) \\
39(70.9) \\
38(69.1)\end{array}$ & & \\
\hline $\begin{array}{l}\text { NIHSS } \\
\begin{array}{l}0 \text { (n [\%]) } \\
\text { I (n [\%]) } \\
2 \text { (n [\%]) } \\
3 \text { (n [\%]) } \\
4 \text { (n [\%]) }\end{array}\end{array}$ & $\begin{array}{l}26(47.3) \\
14(25.5) \\
10(18.2) \\
4(7.3) \\
1(1.8)\end{array}$ & $\begin{array}{l}14(48.3) \\
7(24.1) \\
5(17.2) \\
2(6.9) \\
1(3.4)\end{array}$ & 0.935 \\
\hline $\begin{array}{l}\text { Fazekas PV } \\
0 \text { (n [\%]) } \\
\text { I (n [\%]) } \\
2 \text { (n [\%]) } \\
3 \text { (n [\%]) }\end{array}$ & $\begin{array}{l}1(1.8) \\
26(47.3) \\
19(34.5) \\
9(16.4)\end{array}$ & $\begin{array}{l}0(0) \\
17(58.6) \\
8(27.6) \\
4(13.8)\end{array}$ & 0.661 \\
\hline $\begin{array}{c}\text { Fazekas DWM } \\
0 \text { (n [\%]) } \\
\text { I (n [\%]) } \\
2 \text { (n [\%]) } \\
3(\mathrm{n}[\%])\end{array}$ & $\begin{array}{l}3(5.5) \\
26(47.3) \\
20(36.4) \\
6(10.9)\end{array}$ & $\begin{array}{l}4(13.8) \\
18(62.1) \\
6(20.7) \\
1(3.4)\end{array}$ & 0.063 \\
\hline LDL (mmol/L) & $2.78 \pm 1.08$ & $2.87 \pm 0.82$ & 0.483 \\
\hline $\mathrm{HDL}(\mathrm{mmol} / \mathrm{L})$ & $1.10 \pm 0.23$ & $1.05 \pm 0.26$ & 0.189 \\
\hline
\end{tabular}

(Continued) 
Table 2 (Continued).

\begin{tabular}{|l|l|l|l|}
\hline & $\begin{array}{l}\text { Cognitive } \\
\text { Impairment } \\
\text { Group (n=55) }\end{array}$ & $\begin{array}{l}\text { Non-Cognitive } \\
\text { Impairment } \\
\text { Group n=29) }\end{array}$ & P value \\
\hline HCY (umol/L) & $14.15 \pm 7.92$ & $12.35 \pm 5.26$ & 0.320 \\
\hline UA (umol/L) & $310.93 \pm 78.53$ & $347.35 \pm 89.34$ & 0.079 \\
\hline Vitamin BI2 (pg/mL) & $411.61 \pm 263.40$ & $389.32 \pm 227.22$ & 0.784 \\
\hline
\end{tabular}

Notes: In the cognitive-impairment group, 54 patients underwent low-density lipoprotein (LDL) testing, 53 patients underwent high-density lipoprotein (HDL) testing, 50 underwent homocysteine (HCY) testing, 54 underwent uric acid (UA) testing, and $4 \mathrm{I}$ underwent vitamin $\mathrm{BI} 2$ testing. In the non-cognitive-impairment group, 27 patients underwent LDL and HDL testing, 25 underwent HCY testing, 28 underwent $U A$ testing, and 22 underwent vitamin $B 12$ testing.

Abbreviations: PV, periventricular; DWM, deep white matter; NIHSS, National Institutes of Health Stroke Scale; TOAST, Trial of Org 10172 in acute stroke treatment.

(Groupdiff $<0$ ) in patients with TIA/MIS (Table 6 and Figure 4).

\section{Differences in Differentially Methylated} Sites in Patients with Transient Ischemic Stroke/Mild Ischemic Stroke with and without Cognitive Impairment

RIN3 gene methylation levels were significantly different between patients with TIA/MIS with and without cognitive impairment. We examined methylation levels at different sites between both groups and found statistically significant differences $(P<0.05)$ at several sites (S26113, 165, S27-25, 36, 111, 116, 127, 144, 146, and 152). All of these sites, except site S26-165 (Groupdiff $=0.0019$, $P=0.03$ ), showed hypomethylation in the cognitive impairment group after adjusting for gender, age, education, CHD, and DWM. There groups were not significantly different following adjustment at sites S26-113, 165, S2736, and 127 (Table 7 and Figure 5).

\section{Differences in Differentially Methylated}

\section{Segments Between Patients with}

Transient Ischemic Stroke/Mild Ischemic Stroke and Healthy Controls

We investigated whether there were differences in the methylation levels of different fragments between patients with TIA/MIS and healthy controls. By calculating the mean methylation levels of all CpGs of the RIN3 gene, we found that there were statistically significant differences in RIN3$26(P=0.03)$ and $R I N 3-27(P<0.001)$ between both groups. The overall methylation levels of RIN3-26 (Groupdiff $=$ -0.0011 ) and RIN3-27 (Groupdiff $=-0.0417$ ) were significantly lower in the TIA/MIS group than in the control group. However, after adjusting for gender and age, there was only a statistically significant difference in RIN3-27 between both groups (Adj. $P<0.001$ ) (Table 8).

\section{Differences in Differentially Methylated} Segments Between Patients with TIA/MIS We investigated whether there were differences in the methylation levels of different fragments between the cognitive impairment and non-cognitive impairment TIA/MIS groups. By calculating the mean methylation levels of all CpGs of the RIN3 gene, we found that there was a statistically significant difference in RIN3-27 between both groups ( $P=0.01$ ), even after adjusting for age, gender, education, CHD, and DWM (Adj. $P=0.046$ ) (Table 9). The methylation level of RIN3-27 was significantly lower (Groupdiff $=-0.0268$ ) in the cognitive-impairment group than in the non-cognitive-impairment group.

\section{Predictive Model for Acute-Phase TIA/ MIS with Cognitive Impairment}

After analysing clinical data from the cognitive-impairment and non-cognitive-impairment groups (Table 2) and

Table 3 Information on Target DNA Methylation Sequencing

\begin{tabular}{|c|c|c|c|c|c|c|c|c|c|c|c|}
\hline Target & Chr & Gene & mRNA & $\begin{array}{l}\text { mRNA } \\
\text { Strand }\end{array}$ & TSS & TES & Start & End & Length & $\begin{array}{l}\text { Target } \\
\text { Strand }\end{array}$ & Distance2TSS \\
\hline RIN3-26 & 14 & RIN3 & NM_024832 & + & 9298,012 & 93155339 & 92979633 & 92979850 & 218 & + & -491 \\
\hline RIN3-27 & 14 & RIN3 & NM_024832 & + & 92980124 & 93155339 & 92980930 & 92981104 & 175 & + & 806 \\
\hline
\end{tabular}

Abbreviations: Target, target segment name; Chr, chromosome; Gene, gene name; mRNA, mRNA closer to the product; mRNA strand, mRNA direction; TSS, transcription start site of mRNA; TES, transcriptional end site of mRNA; Start, starting position of the product on reference genomes; End, ending position of the product on reference genomes; Length, length of the product; Target strand, direction of the product; Distance2TSS, relative distance between product; TSS, a negative sign indicates that the site is upwards TSS. 


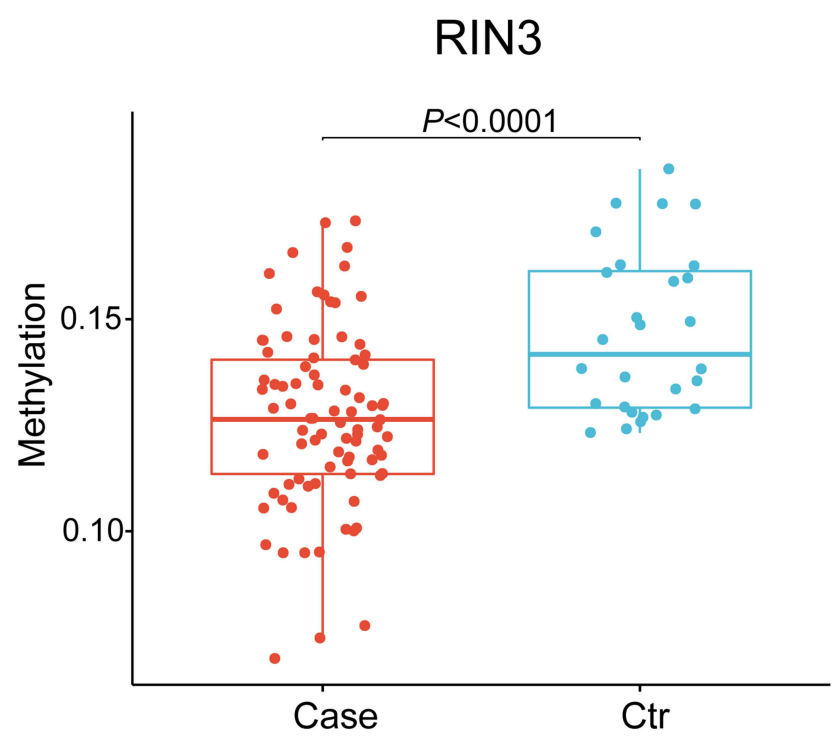

Figure 2 Shows the difference in RIN3 methylation levels between the case group (group J) and the control group (group C). The RIN3 gene was relatively hypomethylated in group J. Students $t$-test was used to compare the groups.

combining this information with statistically significant differences in RIN3 methylation sites, we constructed a predictive model and plotted ROC curves. We found that the combination of three risk factors, including education level, presence of coronary heart disease, and S27-146 site methylation, showed the highest sensitivity/specificity for predicting early cognitive impairment after TIA/MIS. Thus, this model was able to scientifically forecast the occurrence of early cognitive impairment after TIA/MIS, with an area under the curve of 0.808 (95\% CI: 0.7119 0.9044) (Figure 6).

\section{Discussion}

This study demonstrated that hypomethylation of the RIN3 gene was associated with TIA/MIS and with early cognitive impairment after its onset. Furthermore, a combination of clinical indicators and methylation sites could predict the onset of early cognitive impairment after

Table 4 Differences in RIN3 Methylation Levels Between Transient Ischemic Stroke/Mild Ischemic Stroke Patients and Healthy Controls

\begin{tabular}{|l|l|l|l|}
\hline Gene & P value & Groupdiff & Adj. $P$ value \\
\hline RIN3 & $<0.001$ & -0.02 & $<0.001$ \\
\hline
\end{tabular}

Note: Students $t$-test was used to compare the groups.

Abbreviations: Gene, gene name; Groupdiff, difference in mean methylation between the case and control groups; Adj. $P$-value, $P$-value after adjusting for age and gender.

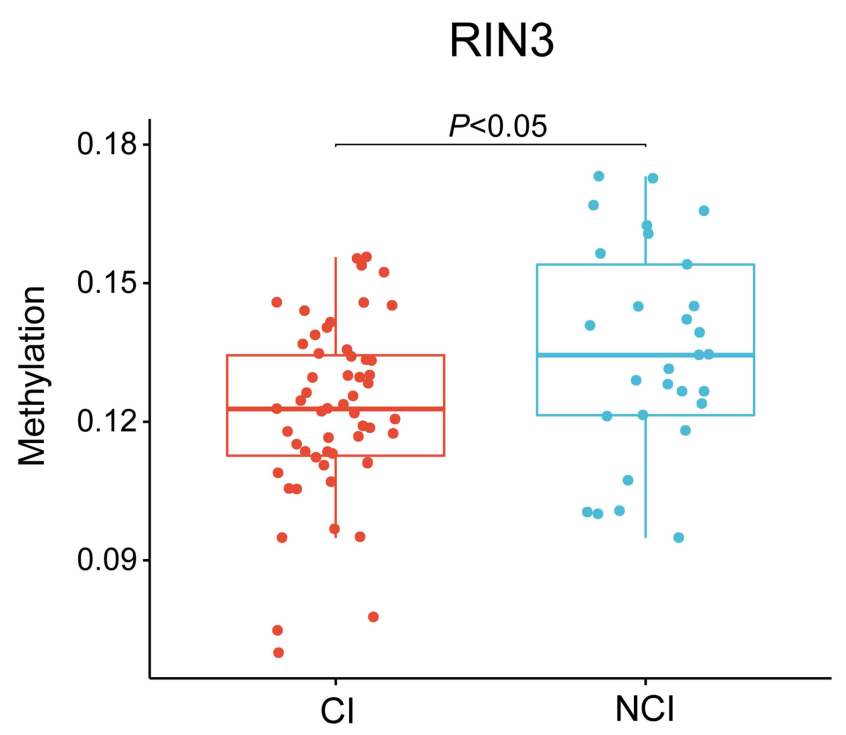

Figure 3 Compares the RIN3 methylation levels between the group with cognitive impairment after TIA/MIS (group A) and the group without cognitive impairment after TIA/MIS (group B). The RIN3 gene was hypomethylated in the former. Students $t$-test was used to compare the groups.

Abbreviations: Ctr, control; $\mathrm{Cl}$, cognitive impairment; $\mathrm{NCl}$, non-cognitive impairment.

TIA/MIS. At present, there are few studies related to the relationship between gene methylation and early cognitive impairment after TIA/MIS, prompting this study.

Currently, there is no standardised scale for measuring vascular cognitive impairment. In this study, we used four scales to detect cognitive impairment in individual domains and found that the incidence of cognitive impairment after TIA/MIS was $65.5 \%(55 / 84)$, which is consistent with results reported in other studies. ${ }^{1,3,4}$ Thus, the results obtained from these scales reflected the cognitive status of patients at that time. We investigated the correlation between RIN3 methylation and TIA/MIS and between RIN3 methylation and TIA/MIS with early cognitive impairment. Our results showed that the RIN3 gene was hypomethylated in the whole blood of patients with TIA/ MIS relative to a healthy control group. In addition, there was hypomethylation of the RIN3 gene in TIA/MIS

Table 5 Differences in RIN3 Methylation Levels Between Patients with Transient Ischemic Stroke/Mild Ischemic Stroke with and without Cognitive Impairment

\begin{tabular}{|l|l|l|l|}
\hline Gene & P value & Groupdiff & Adj.P value \\
\hline RIN3 & 0.01 & -0.013 & 0.044 \\
\hline
\end{tabular}

Note: Students $t$-test was used to compare the groups.

Abbreviations: Gene, gene name; Groupdiff, difference in mean methylation levels between the cognitive impairment and non-cognitive impairment groups; Adj. $P$-value, $P$-value after adjusting for age, gender, education, $C H D$, and DWM; Edu, education; CHD, coronary heart disease; DWM, deep white matter. 
Table 6 Differences in Methylation Sites Between Patients with Transient Ischemic Stroke/Mild Ischemic Stroke and Healthy Controls

\begin{tabular}{|c|c|c|c|c|c|}
\hline Target & POS & Type & Groudiff & $P$ value & Adj.P value \\
\hline$S 26$ & 146 & CG & -0.0024 & 0.008 & 0.007 \\
\hline S27 & 25 & CG & -0.0517 & $<0.001$ & $<0.001$ \\
\hline S27 & 29 & CG & -0.0392 & $<0.001$ & $<0.001$ \\
\hline S27 & 36 & CG & -0.0488 & $<0.001$ & $<0.001$ \\
\hline S27 & 38 & CG & -0.0418 & $<0.001$ & $<0.001$ \\
\hline S27 & 40 & CG & -0.0509 & $<0.001$ & $<0.001$ \\
\hline S27 & 58 & CG & -0.0370 & $<0.001$ & $<0.001$ \\
\hline S27 & 61 & CG & -0.0356 & $<0.001$ & $<0.001$ \\
\hline S27 & 96 & CG & -0.0164 & 0.001 & 0.002 \\
\hline S27 & 111 & CG & -0.0459 & $<0.001$ & 0.003 \\
\hline S27 & 116 & CG & -0.0525 & $<0.001$ & 0.001 \\
\hline S27 & 127 & CG & -0.0373 & $<0.001$ & $<0.001$ \\
\hline S27 & 144 & CG & -0.0374 & 0.001 & 0.008 \\
\hline S27 & 146 & CG & -0.0430 & $<0.001$ & 0.008 \\
\hline S27 & 152 & CG & -0.0465 & $<0.001$ & $<0.001$ \\
\hline
\end{tabular}

Note: Students $t$-test was used to compare the groups.

Abbreviations: Target, site name; POS, position of the methylated site on the fragment; Type, methylation type; Groupdiff, difference in mean methylation between the case and control groups; $P$-value, $P$-value after adjusting for age and gender.

patients with acute-phase cognitive impairment in comparison to TIA/MIS patients without acute-phase cognitive impairment. Shen et al have shown that increased expression levels of RIN3 can affect its transport function, which may lead to an increase in the $\alpha \beta$ protein within neurons. Boden found that patients with early-onset $A D$ showed hypomethylation of the RIN3 gene in whole blood, which led to higher expression of the RIN3 gene. ${ }^{8}$ Another study confirmed that $\alpha \beta$ plays a role in vascular cognitive impairment. ${ }^{19}$ Therefore, we hypothesized that patients with TIA/MIS would also show abnormal methylation of the RIN3 gene.

Cognitive impairment is common within 7 days after onset of TIA/MIS and may persist even after recovery of motor symptoms, leading to a greater risk of cognitive impairment and a reduced cognitive reserve in the distant future. Despite the rapid recovery of motor symptoms in TIA/MIS patients, cognitive impairment can easily be overlooked in these patients. ${ }^{2}$ The occurrence of early cognitive impairment after TIA/MIS is influenced by multiple factors, and the current study found that some imaging changes may serve as predictors. Suda et al found that temporal horn atrophy on MRI education and smoking are independent risk factors for cognitive impairment. ${ }^{1}$ Takahashi also confirmed that moderate temporal lobe atrophy on MRA source images combined with less years of education were predictors of cognitive impairment in the acute phase of ischemic stroke. ${ }^{20}$ Finally, Zamboni found that white-matter brain damage on MRI was associated with TIA/MIS with early cognitive impairment damage. ${ }^{21}$ These factors are, to some extent, nonmodifiable. Furthermore, it can be difficult to standardise their quantitative detection criteria. To date, there has been a lack of identification of modifiable biomolecular indicators related to disease onset, as well as a lack of quantifiable blood molecular indicators. This study, however, found that the RIN3 gene and abnormal methylation of sites on this gene are associated with early cognitive impairment after TIA/MIS. Since methylation levels can be affected by environmental factors and can be measured quantitatively, they may be useful for the design of potential interventions and predictive models.

There is an increasing number of studies related to the correlation between methylation and ischemic stroke. ${ }^{22-28}$ For example, methylation of the $A B C G 1$ and $A P O E$ genes has been correlated with cerebral infarction and atherosclerosis, ${ }^{23}$ while MMP-2, LINE3 and TP53 methylation has been correlated with ischemic stroke, ${ }^{24-26}$ and CDKN2B has been correlated with arterial calcification in ischemic stroke. ${ }^{27,28}$ Furthermore, methylation has been strongly correlated with the pathogenesis of ischemic stroke. However, these studies have mainly focused on the relationship between gene methylation and ischemic stroke or atherosclerosis rather than specifically focusing on TIA/MIS. Moreover, there have been few studies related to the relationship between methylation and early cognitive impairment after stroke.

Some studies have investigated molecular indicators in the cerebrospinal fluid of patients with vascular dementia (VD) or VCI and have found that $\mathrm{A} \beta 42$ are significantly decreased in the cerebrospinal fluid of patients with VCI compared to controls. ${ }^{29-33} \mathrm{~A} \beta$ concentration in red blood cell of VD patients is higher than that of controls. ${ }^{34}$ Elevated $A \beta$ levels in blood may aggravate vascular amyloidosis and thus affect cognition. ${ }^{35}$ However, whether $A \beta$ in blood can predict VCI still requires verification. NF- $\mathrm{KB}$ and VEGF levels have also been shown to be elevated in the cerebrospinal fluid of patients with VD, which may affect the $\beta$-amyloid protein. ${ }^{36,37}$ Abnormal expression of the $A \beta$ protein has been proposed to be significantly associated with vascular-related cognitive impairment. 


\section{RIN3}

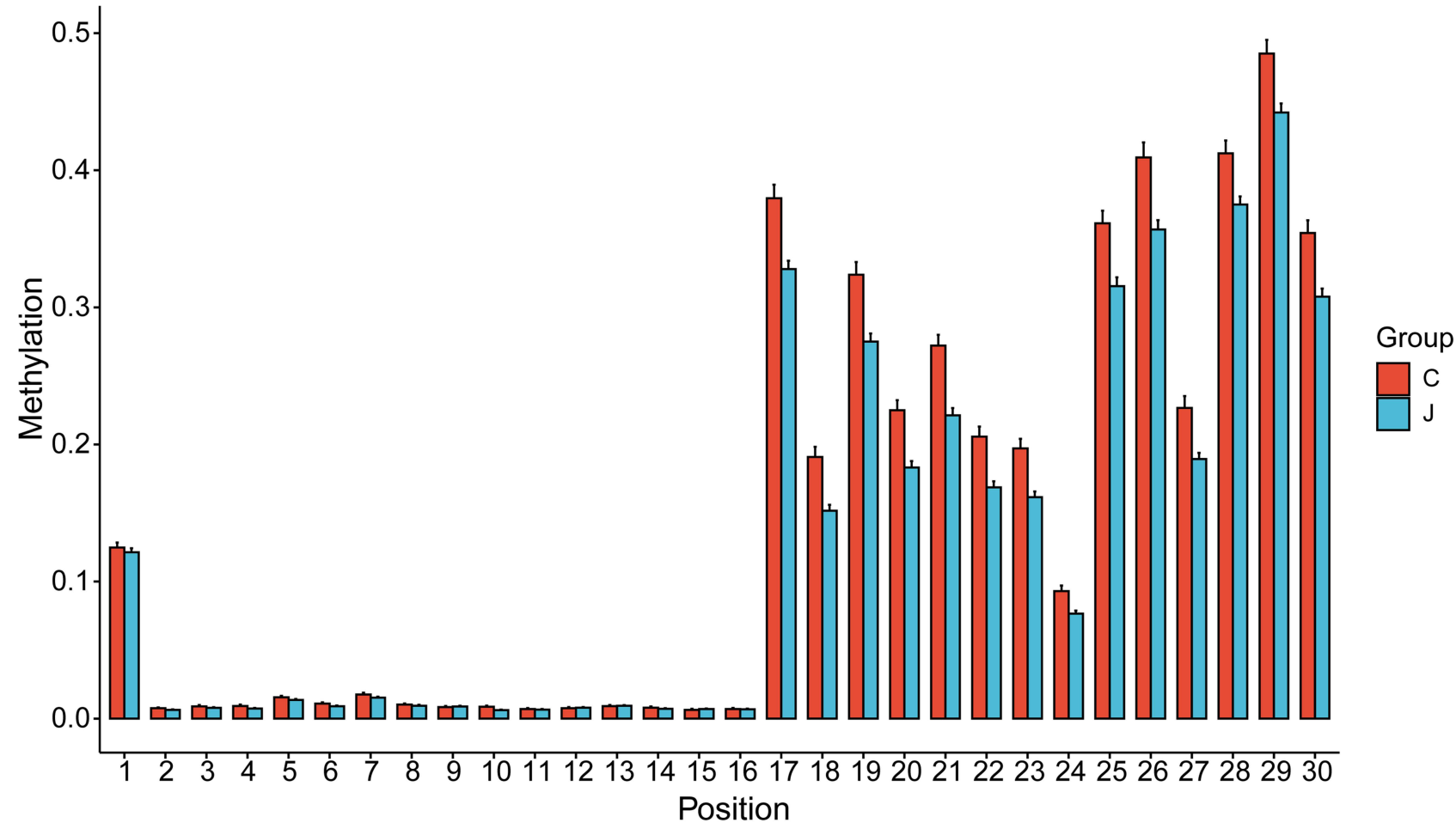

Figure 4 Compares methylation levels at measured sites in the case group (group J) and control group (group C), with the X-axis indicating the detected site and the Y-axis indicating the methylation level at each site. Students $t$-test was used to compare the groups.

The RIN3 gene located on chromosome 14, however, has been less well studied. This gene has, however, been found to affect endocytosis of axonemes by interacting with BIN1, which influences the transport and metabolism

Table 7 Different Methylation Sites in Patients with Transient Ischemic Stroke/Mild Ischemic Stroke with and without Cognitive Impairment

\begin{tabular}{|l|l|l|l|l|l|}
\hline Target & POS & Type & Groudiff & $P$ value & Adj.P value \\
\hline S26 & 113 & CG & -0.0033 & 0.02 & 0.16 \\
S26 & 165 & CG & 0.0019 & 0.03 & 0.09 \\
S27 & 25 & CG & -0.0369 & 0.006 & 0.03 \\
S27 & 36 & CG & -0.0282 & 0.03 & 0.08 \\
S27 & III & CG & -0.0433 & 0.002 & 0.01 \\
S27 & 116 & CG & -0.0468 & 0.002 & 0.01 \\
S27 & 127 & CG & -0.0206 & 0.03 & 0.08 \\
S27 & 144 & CG & -0.0380 & 0.003 & 0.02 \\
S27 & 146 & CG & -0.0476 & 0.001 & 0.007 \\
S27 & 152 & CG & -0.0370 & 0.005 & 0.02 \\
\hline
\end{tabular}

Note: Students $t$-test was used to compare the groups.

Abbreviations: Target, site name; POS, position of the methylated site on the fragment; Type, methylation type; Groupdiff, difference in mean methylation between both groups (patients with and without cognitive impairment); Adj. $P$-value, $P$-value after correction for age, gender, education, CHD, and DWM; Edu, education; CHD, coronary heart disease; DWM, deep white matter. of the $\mathrm{A} \beta$ protein, with high expression of the RIN3 gene decreasing $A \beta$ protein metabolism. ${ }^{5,6}$ It has also been shown that abnormal RIN3 gene expression is associated with $\mathrm{AD}^{7}$ and that whole-blood RIN3 methylation levels are low in $\mathrm{AD}$ patients. ${ }^{8}$ RIN3 affects cognitive function mainly by influencing $A \beta$ protein transport. It is therefore likely that the appearance of early cognitive impairment in TIA/MIS patients is also associated with abnormal RIN3 gene expression and with altered $A \beta$ protein levels. The methylation level is an important factor affecting gene expression. This study confirmed that abnormal RIN3 gene methylation was associated with early cognitive impairment in TIA/MIS.

This study examined RIN3 methylation levels in whole blood and found that the RIN3 gene was hypomethylated in the TIA/MIS group compared to the control group and that RIN3 methylation levels were lower in TIA/MIS patients with early cognitive impairment than in those without cognitive impairment. We hypothesize that RIN3 hypomethylation occurs during the acute phase of TIA and mild stroke, which, in turn, leads to an abnormal accumulation of the $A \beta$ protein in the blood. Indeed, this study did find a correlation between abnormal RIN3 methylation in 


\section{RIN3}

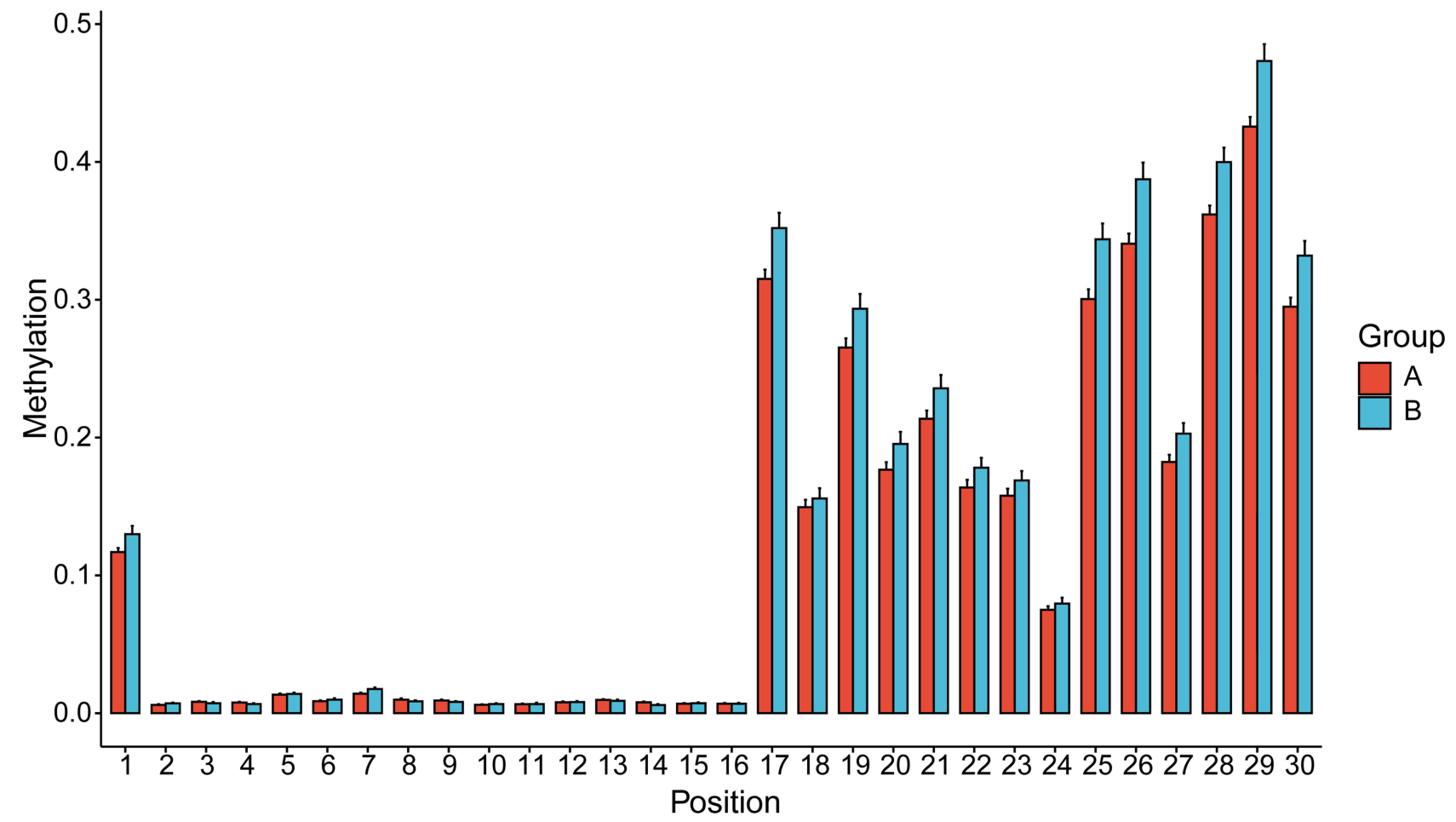

Figure 5 Compares methylation levels at measured sites between the group with cognitive impairment after TIA/MIS (group A) and the group without cognitive impairment after TIA/MIS (group B), with the X-axis indicating the detected site and the Y-axis indicating the methylation level. Students $t$-test was used to compare the groups.

blood and TIA/MIS with early cognitive impairment; however, the causal relationship between the two remains unclear. Based on our study, it is not possible to determine whether RIN3 gene hypomethylation in the blood led to an accumulation of the $A \beta$ protein, which resulted in the appearance or exacerbation of symptoms, or whether the disease itself first affected the level of RIN3 methylation, which in turn affected the A $\beta$ protein level in blood. These issues require further research.

This study had some limitations. First, the number of cases in this study was small and not fully matched by age nor sex. However, we corrected for age and sex to minimise the influence of these factors on our statistical analyses.

Table 8 Differences in Differentially Methylated Segments Between Patients with Transient Ischemic Stroke/Mild Ischemic Stroke and Healthy Controls

\begin{tabular}{|l|l|l|l|}
\hline Target & Groupdiff & P value & Adj.P value \\
\hline RIN3-26 & -0.0011 & 0.03 & 0.17 \\
RIN3-27 & -0.0417 & $<0.001$ & $<0.001$ \\
\hline
\end{tabular}

Note: Students $t$-test was used to compare the groups.

Abbreviations: Target, segment name; Groupdiff, difference in mean methylation between the case and control groups; Adj. $P$-value, $P$-value after adjusting for age and gender.
Second, this study was designed to explore biomarkers in whole blood, so we examined DNA methylation in whole blood. However, we cannot confirm whether methylation levels in whole blood are reflected in methylation levels in brain tissue. However, we chose to test whole blood because the underlying cause of cerebrovascular disease involves changes to the blood vessels and the blood itself, which, in turn, leads to brain-tissue damage.

\section{Conclusion}

This study confirmed that there was hypomethylation of the RIN3 gene in the whole blood of TIA/MIS patients relative to controls. Furthermore, there was hypomethylation of the

Table 9 Differences in Differentially Methylated Segments Between Patients with Transient Ischemic Stroke/Mild Ischemic Stroke with and without Cognitive Impairment

\begin{tabular}{|l|l|l|l|}
\hline Target & Groupdiff & $P$ value & Adj.P value \\
\hline RIN3-27 & -0.0268 & 0.01 & 0.046 \\
\hline
\end{tabular}

Note: Students $t$-test was used to compare the groups.

Abbreviations: Target, segment name; Groupdiff, difference in mean methylation between the cognitive impairment and non-cognitive impairment groups; Adj. $P$-value, $P$-value after adjusting for age, gender, education, CHD, and DWM; Edu, education; CHD, coronary heart disease; DWM, deep white matter. 


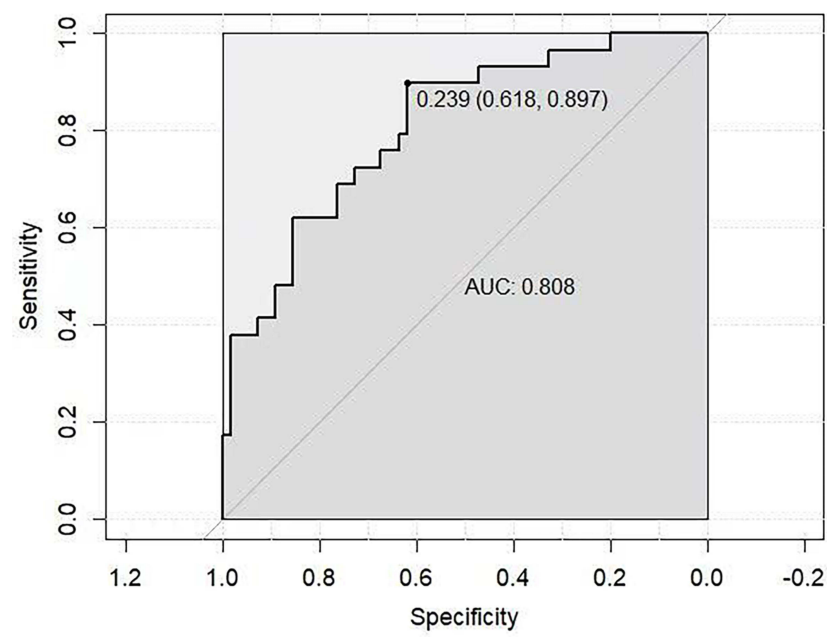

Figure 6 Showed the sensitivity/specificity for predicting early cognitive impairment after TIA/MIS.

RIN3 gene in the whole blood of patients with early cognitive impairment after TIA/MIS relative to patients without early cognitive impairment after TIA/MIS. Therefore, epigenetic modification of the RIN3 gene was strongly associated with TIA/MIS and with TIA/MIS with early cognitive impairment. Based on the modifiable nature of methylation, our study suggests that it may be possible to influence this disease process by methylation via appropriate lifestyle and/ or clinical interventions. Furthermore, since methylation can be quantified and easily analysed, the methylation levels of gene sites combined with clinical information can predict the occurrence of TIA/MIS with early cognitive impairment.

\section{Data Sharing Statement}

Datasets and codes of methylation used in the analyses are stored by the first author and will be provided upon request.

\section{Ethics}

This study was approved by the Ethics Committee of the Qilu Hospital of Shandong University (Qingdao). All patients provided written informed consent. The study complies with the Declaration of Helsinki.

\section{Acknowledgments}

This work was supported by Qingdao Key Health Discipline Development Fund and Scientific Research Foundation of Qilu Hospital of Shandong University (Qingdao) (No.QDKY2019ZD02)

\section{Author Contributions}

All authors made a significant contribution to the work reported, whether that is in the conception, study design, execution, acquisition of data, analysis and interpretation, or in all these areas; took part in drafting, revising or critically reviewing the article; gave final approval of the version to be published; have agreed on the journal to which the article has been submitted; and agree to be accountable for all aspects of the work.

\section{Disclosure}

The authors declare that there is no conflicts of interest or financial relationships that could be construed as a potential conflict of interest in this work.

\section{References}

1. Suda S, Nishimura T, Ishiwata A, et al. Early cognitive impairment after minor stroke: associated factors and functional outcome. J Stroke Cerebrovasc Dis. 2020;29(5):104749. doi:10.1016/j. jstrokecerebrovasdis.2020.104749

2. Pendlebury ST, Wadling S, Silver LE, Mehta Z, Rothwell PM. Transient cognitive impairment in TIA and minor stroke. Stroke. 2011;42(11):3116-3121. doi:10.1161/STROKEAHA.111.621490

3. Blackburn DJ, Bafadhel L, Randall M, Harkness KA. Cognitive screening in the acute stroke setting. Age Ageing. 2013;42 (1):113-116. doi:10.1093/ageing/afs116

4. Godefroy O, Fickl A, Roussel M, et al. Is the montreal cognitive assessment superior to the mini-mental state examination to detect poststroke cognitive impairment? A study with neuropsychological evaluation. Stroke. 2011;42(6):1712-1716. doi:10.1161/ STROKEAHA.110.606277

5. Kajiho H, Saito K, Tsujita K, et al. RIN3: a novel Rab5 GEF interacting with amphiphysin II involved in the early endocytic pathway. $J$ Cell Sci. 2003;116(Pt20):4159-4168. doi:10.1242/ jcs.00718

6. Kajiho H, Sakurai K, Minoda T, et al. Characterization of RIN3 as a guanine nucleotide exchange factor for the Rab5 subfamily GTPase Rab31. J Biol Chem. 2011;286(27):24364-24373. doi:10.1074/jbc. M110.172445

7. Shen R, Zhao X, He L, et al. Upregulation of RIN3 induces endosomal dysfunction in Alzheimer's disease. Transl Neurodegener. 2020;9(1):26. doi:10.1186/s40035-020-00206-1

8. Boden KA, Barber IS, Clement N, et al. Methylation profiling RIN3 and MEF2C identifies epigenetic marks associated with sporadic early onset alzheimer's disease. J Alzheimers Dis Rep. 2017;1 (1):97-108. doi:10.3233/ADR-170015

9. Tian JZ, Xie HG, Qin B, et al. Screening and diagnostic framework of vascular dementia in Chinese population. Zhonghua Nei Ke Za Zhi. 2019;58(1):10-16. doi:10.3760/cma.j.issn.05781426.2019.01.003

10. Hao L, Xing Y, Li X, et al. Risk factors and neuropsychological assessments of subjective cognitive decline (plus) in chinese memory clinic. Front Neurosci. 2019;13:846. doi:10.3389/fnins.2019.00846

11. Ma J, Zhang Y, Guo Q. Comparison of vascular cognitive impairment-no dementia by multiple classification methods. Int $J$ Neurosci. 2015;125(11):823-830. doi:10.3109/00207454.2014.972504 
12. Xu Y, Chen K, Zhao Q, Li F, Guo Q. Short-term delayed recall of auditory verbal learning test provides equivalent value to long-term delayed recall in predicting MCI clinical outcomes: a longitudinal follow-up study. Appl Neuropsychol Adult. 2020;27(1):73-81. doi:10.1080/23279095.2018.1481067(AVLT

13. Llinàs-Reglà $\mathrm{J}$, Vilalta-Franch $\mathrm{J}$, López-Pousa $\mathrm{S}$, Calvó-Perxas L, Torrents Rodas D, Garre-Olmo J. The trail making test. Assessment. 2017;24(2):183-196. doi:10.1177/1073191115602552

14. Sánchez-Cubillo I, Periáñez JA, Adrover-Roig D, et al. Construct validity of the trail making test: role of task-switching, working memory, inhibition/interference control, and visuomotor abilities. $J$ Int Neuropsychol Soc. 2009;15(3):438-450. doi:10.1017/ S1355617709090626

15. Wei M, Shi J, Li T, et al. Diagnostic accuracy of the chinese version of the trail-making test for screening cognitive impairment. $J \mathrm{Am}$ Geriatr Soc. 2018;66(1):92-99. doi:10.1111/jgs.15135

16. Gorelick PB, Counts SE, Nyenhuis D. Vascular cognitive impairment and dementia. Biochim Biophys Acta. 2016;1862(5):860-868. doi:10.1016/j.bbadis.2015.12.015

17. Magoč T, Salzberg SL. FLASH: fast length adjustment of short reads to improve genome assemblies. Bioinformatics. 2011;27 (21):2957-2963. doi:10.1093/bioinformatics/btr507

18. Camacho C, Coulouris G, Avagyan V, et al. BLAST+: architecture and applications. BMC Bioinform. 2009;10:421. doi:10.1186/14712105-10-421

19. Cipollini V, Troili F, Giubilei F. Emerging biomarkers in vascular cognitive impairment and dementia: from pathophysiological pathways to clinical application. Int J Mol Sci. 2019;20(11):2812. doi:10.3390/ijms20112812

20. Takahashi Y, Saito S, Yamamoto Y, et al. Visually-rated medial temporal lobe atrophy with lower educational history as a quick indicator of amnestic cognitive impairment after stroke. J Alzheimers Dis. 2019;67(2):621-629. doi:10.3233/JAD-180976

21. Zamboni G, Griffanti L, Jenkinson M, et al. White matter imaging correlates of early cognitive impairment detected by the montreal cognitive assessment after transient ischemic attack and minor stroke. Stroke. 2017;48(6):1539-1547. doi:10.1161/ STROKEAHA.116.016044

22. Deng GX, Xu N, Huang Q, et al. Association between promoter DNA methylation and gene expression in the pathogenesis of ischemic stroke. Aging (Albany NY). 2019;11(18):7663-7677. doi:10.18632/aging. 102278

23. Qin X, Li J, Wu T, et al. Overall and sex-specific associations between methylation of the ABCG1 and APOE genes and ischemic stroke or other atherosclerosis-related traits in a sibling study of Chinese population. Clin Epigenetics. 2019;11(1):189. doi:10.1186/ s13148-019-0784-0

24. Lin HF, Hsi E, Huang LC, et al. Methylation in the matrix metalloproteinase-2 gene is associated with cerebral ischemic stroke. J Investig Med. 2017;65(4):794-799. doi:10.1136/jim-2016000277

Neuropsychiatric Disease and Treatment

\section{Publish your work in this journal}

Neuropsychiatric Disease and Treatment is an international, peerreviewed journal of clinical therapeutics and pharmacology focusing on concise rapid reporting of clinical or pre-clinical studies on a range of neuropsychiatric and neurological disorders. This journal is indexed on PubMed Central, the 'PsycINFO' database and CAS, and
25. Wei Y, Sun Z, Wang Y, et al. Methylation in the TP53 promoter is associated with ischemic stroke. Mol Med Rep. 2019;20 (2):1404-1410. doi:10.3892/mmr.2019.10348

26. Lin RT, Hsi E, Lin HF, Liao YC, Wang YS, Juo SH. LINE-1 methylation is associated with an increased risk of ischemic stroke in men. Curr Neurovasc Res. 2014;11(1):4-9. doi:10.2174/ 1567202610666131202145530

27. Zhou S, Zhang Y, Wang L, et al. CDKN2B methylation is associated with carotid artery calcification in ischemic stroke patients. $J$ Transl Med. 2016;14:333. doi:10.1186/s12967-016-1093-4

28. Zhou S, Cai B, Zhang Z, et al. CDKN2B methylation and aortic arch calcification in patients with ischemic stroke. J Atheroscler Thromb. 2017;24(6):609-620. doi:10.5551/jat.36897

29. Llorens F, Schmitz M, Knipper T, et al. Cerebrospinal fluid biomarkers of alzheimer's disease show different but partially overlapping profile compared to vascular dementia. Front Aging Neurosci. 2017;9:289. doi:10.3389/fnagi.2017.00289

30. Ewers M, Mattsson N, Minthon L, et al. CSF biomarkers for the differential diagnosis of Alzheimer's disease: a large-scale international multicenter study. Alzheimers Dement. 2015;11 (11):1306-1315. doi:10.1016/j.jalz.2014.12.006

31. Struyfs H, Van Broeck B, Timmers M, et al. Diagnostic accuracy of cerebrospinal fluid amyloid- $\beta$ isoforms for early and differential dementia diagnosis. $J$ Alzheimers Dis. 2015;45(3):813-822. doi:10.3233/JAD-141986

32. Duits FH, Hernandez-Guillamon M, Montaner J, et al. Matrix metalloproteinases in alzheimer's disease and concurrent cerebral microbleeds. J Alzheimers Dis. 2015;48(3):711-720. doi:10.3233/JAD143186

33. Horvath I, Jia X, Johansson P, et al. Pro-inflammatory S100A9 protein as a robust biomarker differentiating early stages of cognitive impairment in alzheimer's disease. ACS Chem Neurosci. 2016;7 (1):34-39. doi:10.1021/acschemneuro.5b00265

34. Lauriola M, Paroni G, Ciccone F, et al. Erythrocyte associated amyloid- $\beta$ as potential biomarker to diagnose dementia. Curr Alzheimer Res. 2018;15(4):381-385. doi:10.2174/ 1567205014666171110160556

35. Yamada M. Cerebral amyloid angiopathy: emerging concepts. J Stroke. 2015;17(1):17-30. doi:10.5853/jos.2015.17.1.17

36. Buchhave P, Zetterberg H, Blennow K, Minthon L, Janciauskiene S, Hansson O. Soluble TNF receptors are associated with A $\beta$ metabolism and conversion to dementia in subjects with mild cognitive impairment. Neurobiol Aging. 2010;31(11):1877-1884. doi:10.1016/ j.neurobiolaging.2008.10.012

37. Tarkowski E, Issa R, Sjögren M, et al. Increased intrathecal levels of the angiogenic factors VEGF and TGF-beta in Alzheimer's disease and vascular dementia. Neurobiol Aging. 2002;23(2):237-243. doi:10.1016/s0197-4580(01)00285-8 is the official journal of The International Neuropsychiatric Association (INA). The manuscript management system is completely online and includes a very quick and fair peer-review system, which is all easy to use. Visit http://www.dovepress.com/testimonials.php to read real quotes from published authors.

\section{Dovepress}

